\title{
Changes in Australian Early-Career General Practitioners' Benzodiazepine Prescribing: a Longitudinal Analysis
}

\author{
Parker Magin, PhD, FRACGP ${ }^{1,2}$, Amanda Tapley, MMedStats ${ }^{1,2}$, Adrian J Dunlop, PhD, FAChAM ${ }^{1,3}$, \\ Andrew Davey, MClinEpid, FRACGP',2, Mieke van Driel, PhD, FRACGP4, Elizabeth Holliday, PhD ${ }^{1,5}$, \\ Simon Morgan, MPH, FRACGP ${ }^{2}$, Kim Henderson, GDipMedSC(HSOCSC) $)^{1,2}$, Jean Ball, GDipMedStats ${ }^{5}$, \\ Nigel Catzikiris, BHIth ${ }^{1,2}$, Katie Mulquiney, BND(Hons) ${ }^{1,2}$, Neil Spike, FRACGP ${ }^{6,7}$, Rohan Kerr, FRACGP ${ }^{8}$, \\ and Simon Holliday, FAChAM ${ }^{7,3}$
}

\begin{abstract}
'School of Medicine and Public Health, University of Newcastle, Callaghan, Australia; ${ }^{2}$ GP Synergy General Practice Regional Training Organisation, Newcastle, Australia; ${ }^{3}$ Drug and Alcohol Clinical Services, Hunter New England Health, Newcastle, Australia; ${ }^{4}$ Faculty of Medicine, University of Queensland, Brisbane, Australia; ${ }^{5}$ Hunter Medical Research Institute, Newcastle, Australia; ${ }^{6}$ Eastern Victoria GP Training, Melbourne, Australia; ${ }^{7}$ University of Melbourne, Melbourne, Australia; ${ }^{8}$ General Practice Training Tasmania, Hobart, Australia.
\end{abstract}

BACKGROUND: Australian and international guidelines recommend benzodiazepines and related drugs (hereafter "benzodiazepines") as second-line, short-term medications only. Most benzodiazepines are prescribed by general practitioners (GPs; family physicians). Australian GP registrars ("trainees" or "residents" participating in a posthospital training, apprenticeship-like, practice-based vocational training program), like senior GPs, prescribe benzodiazepines at high rates. Education within a training program, and experience in general practice, would be expected to reduce benzodiazepine prescribing.

OBJECTIVE: To establish if registrars' prescribing of benzodiazepines decreases with time within a GP training program

DESIGN: Longitudinal analysis from the Registrar Clinical Encounters in Training multi-site cohort study

PARTICIPANTS: Registrars of five of Australia's $17 \mathrm{Re}-$ gional Training Providers. Analyses were restricted to patients $\geq 16$ years.

MAIN MEASURES: The main outcome factor was prescription of a benzodiazepine. Conditional logistic regression was used, with registrar included as a fixed effect, to assess within-registrar changes in benzodiazepineprescribing rates. The "time" predictor variable was "training term" (6-month duration Terms 1-4). To contextualize these "within-registrar" changes, a mixed effects logistic regression model was used, including a random effect for registrar, to assess within-program changes in benzodiazepine-prescribing rates over time. The "time" predictor variable was "year" (2010-2015).

KEY RESULTS: Over 12 terms of data collection, 20102015, 1161 registrars (response rate 96\%) provided data

Prior Presentations Results from this analysis were presented at the Australian General Practice Training and Education Conference. Sydney, Australia Auaust 2017

Electronic supplementary material The online version of this article (https://doi.org/10.1007/s11606-018-4577-5) contains supplementary material which is available to authorized users.

Received October 24, 2017

Revised May 18, 2018

Accepted July 2, 2018

Published online July 23, 2018 on 136,809 face-to-face office-based consultations. Two thousand six hundred thirty-two benzodiazepines were prescribed (for $1.2 \%$ of all problems managed). In the multivariable model, there was a significant reduction in within-program benzodiazepine prescribing over time (year) $(p=<0.001, \mathrm{OR}=0.94, \mathrm{CI}=0.90,0.97)$. However, there was no significant change in 'within-registrar' prescribing over time (registrar Term) $(p=0.92$, OR $=1.00$ [95\% CI = 0.94-1.06]).

CONCLUSIONS: Despite a welcome temporal trend for reductions in overall benzodiazepine prescribing from 2010 to 2015, there is still room for improvement and our findings suggest a lack of effect of specific GP vocational training program education and, thus, an opportunity for targeted education.

KEY WORDS: benzodiazepines; family practice; education, medical, graduate; inappropriate prescribing; deprescriptions

J Gen Intern Med 33(10):1676-84

DOI: $10.1007 / \mathrm{s} 11606-018-4577-5$

(c) Society of General Internal Medicine 2018

\section{INTRODUCTION}

The prescription of benzodiazepines and related drugs including the z-drugs such as zopiclone and zolpidem (hereafter referred to as "benzodiazepines") for long-term use is very common in general practice (family medicine) worldwide. ${ }^{1}$ While there are limited indications for use (e.g., alcohol withdrawal), benzodiazepines are used mainly as hypnotics or anxiolytics or for "off-label" indications. ${ }^{1}$ Current Australian clinical guidelines recommend strongly against benzodiazepine use for anxiety (except "in exceptional circumstances"). ${ }^{2}$ American chronic insomnia guidelines advise cognitive behavior therapy and discourage benzodiazepine use (as strictly second-line short-term use, if at all). ${ }^{3}$

The rationale for benzodiazepine avoidance is principally benzodiazepine use being associated with Alzheimer's disease, worsening insomnia, falls, fractures, adverse drug 
interactions, pneumonia and other respiratory effects, deliberate overdose, increased mortality, and dependence. ${ }^{4-9}$ Physical dependence and withdrawal symptoms are associated with as little as 3-6-week use. ${ }^{9}$ An authoritative systematic review found benzodiazepines to be associated with markedly increased mortality. ${ }^{10}$ Furthermore, the evidence for hypnotic efficacy of benzodiazepines is unconvincing. ${ }^{11}$ A particular concern is the increasing prevalence of, and overdoses attributable to, concomitant benzodiazepine-opioid use. ${ }^{12-15}$

Despite this, benzodiazepines are commonly prescribed. During 2013 in British Columbia, Canada, 14.6\% of women and $8.8 \%$ of men had at least one benzodiazepine prescription. ${ }^{16}$ In a Massachusetts primary care practice-based research network, $15 \%$ of patients received at least one benzodiazepine over a year. ${ }^{17}$ Benzodiazepines are particularly problematic, ${ }^{18}$ and the drug class most commonly classified as potentially inappropriate, for older persons. ${ }^{19}$ Yet benzodiazepines are used by at least $17 \%$ of community-dwelling older Australians. $^{20}$

Prescribing patterns established in general practice can be long-lasting. ${ }^{21}$ In a previous cross-sectional analysis of GP registrars' ("trainees" or "residents") prescribing, we found benzodiazepines were prescribed in $2.1 \%$ of all registrar consultations and comprised $2.2 \%$ of all registrar prescriptions. ${ }^{22}$ They were prescribed in half of instances for either insomnia (28.2\% of instances of benzodiazepine prescribing) or anxiety (21.8\%), and in $0.7 \%$ for alcohol withdrawal, but also for many "off-label" indications. These data also demonstrated that registrars, inconsistent with guideline recommendations, prescribed benzodiazepines mainly as maintenance therapy, to patients with whom they were unfamiliar and to older patients. $^{22}$

In the current analysis, we sought to establish whether prescribing (and initiation) of benzodiazepines changes with early-career experience in general practice. We hypothesized that time (that is, increasing clinical experience) in a primary care setting as well as exposure to a clinical educational program would result in a reduction in benzodiazepine prescribing and benzodiazepine initiation by general practice registrars. In order to contextualize this analysis of "withinregistrar" changes, we also aimed to analyze temporal changes in overall benzodiazepine prescribing within the general practice training program in which the registrars trained ("withinprogram" changes).

\section{METHODS}

This was a longitudinal analysis of 12 rounds of data collection (2010-2015) from the Registrar Clinical Encounters in Training (ReCEnT) study.

\section{Setting and Participants}

Australian GP registrars' 3-year duration training program includes three compulsory 6-month general practice-based terms. $^{23}$ These terms are completed within an apprenticeshiplike model under individual experienced GP's supervision ${ }^{24,} 25$ in small, mostly private, often geographically dispersed, general practices. The apprenticeship-based training is supplemented by regional training provider (RTP)-organized educational sessions $^{24}$ (a minimum 125-h face-to-face over the three terms) plus online education, informed by national curricula. $^{26}$

\section{ReCEnT}

ReCEnT is an ongoing, multicenter cohort study of GP registrars' in-consultation clinical and educational experience. Prior to 2016, it was conducted in five of Australia's 17 RTPs. Geographically, the five RTPs encompassed urban, rural, remote, and very remote practices in five of Australia's six states. Individual RTPs contributed four to 12 rounds of data depending on their commencement in the project. ReCEnT documents GP registrars' in-practice clinical experiences and behaviors, including prescribing.

The ReCEnT methodology has been described previously. $^{27}$ Briefly, registrars complete paper-based case report forms (CRFs) recording details of 60 consecutive consultations at approximately the midpoint of each of their three compulsory general practice training terms. Registrars at one of the five RTPs also collected data during an optional fourth training term. For each patient consultation (that is, for 60 consultations per training term) patient demographics, clinical details and educational actions (see below) are recorded on the CRF. Registrar and practice demographics are documented each collection period via a registrar-completed questionnaire. The longitudinal methodology facilitates exploration of the epidemiology of registrars' practice and evaluation of the effects of educational interventions.

\section{Outcome Variable}

The primary outcome factor was whether a benzodiazepine was prescribed. Benzodiazepines were defined according to the Anatomic Therapeutic Chemical (ATC) Classification codes: N05BA (benzodiazepine derivatives), N05BE (azaspirodecanedione derivatives), N05BX (other anxiolytics), N05CD (benzodiazepine derivatives), N05CF (benzodiazepine-related drugs), N05CM (other hypnotics and sedatives), N05CX (hypnotics and sedatives in combination, excl. barbiturates). We did not include melatonin in our analyses as use of melatonin (while it is commonly used as a hypnotic) has very different implications to benzodiazepines, including a comparatively benign adverseeffect $^{\text {profile. }}{ }^{28}$

The secondary outcome factor was whether a benzodiazepine was initiated (that is, prescribed as a new medication). This secondary outcome recognized that it may be less difficult for a registrar to abstain from initiating a benzodiazepine than to decline to prescribe a continuing 
benzodiazepine previously prescribed by another GP in the practice or a specialist.

\section{Independent Variables}

The main independent variable of interest was time. The measure of time differed for the within-registrar analysis and for the analysis of temporal changes within the training program - the within-program analysis (see below).

Registrar variables were age, gender, country of primary medical graduation (Australian or non-Australian), and the RTP enrolled with.

Patient variables were age, gender, Aboriginal or Torres Strait Islander (Indigenous) status, non-English-speaking background (NESB) status, the patient being new to the practice, and the patient being new to the registrar.

Practice variables were rurality classification (major city, inner regional, outer regional, remote, very remote), socioeconomic index of the practice location, and billing policy (does the practice routinely bulk bill? That is, provide consultations reimbursed solely by government rebate, with no cost to the patient). Practice postcode was used to define the Australian Standard Geographical Classification-Remoteness Area (ASGC-RA) classification (the degree of rurality) of the practice location, ${ }^{29}$ and to define the practice location's Socioeconomic Index for Area (SEIFA) Relative Index of Disadvantage decile. ${ }^{30}$

Consultation variables were duration, whether the problem/ diagnosis was new, whether the problem/diagnosis was a chronic disease, ${ }^{31}$ and whether the registrar sought inconsultation advice or information (from their supervisor or other resources, such as specialists, books, or electronic resources).

Problems/diagnoses addressed in the consultation were coded according to the International Primary Care Classification (ICPC-2). ${ }^{32}$

\section{Statistical Analyses}

The unit of analysis was individual problem/diagnosis managed, rather than consultation. Analyses were limited to consultations with patients 16 years or older.

The proportion of problems/diagnoses in which a benzodiazepine was prescribed was calculated with $95 \%$ confidence intervals (95\% CIs), adjusted for clustering within registrars. The proportion of problems/diagnoses (excluding those for which a "continuing" prescription for benzodiazepine was made) for which a benzodiazepine was initiated was also calculated.

Temporal Changes in Benzodiazepine Prescribing and Initiation Within the Training Program ("Within-Program"). The variable "year" has been used as the time component for this analysis. "Year" took values from 2010 to 2015, inclusive. Year has been included as a continuous variable in all analyses, but is presented in the demographics tables as frequencies and percentages by year.
A mixed effects logistic regression model was used, including a random effect for registrar to account for repeated measures on registrars over time. In this model, the parameter estimate for the time variable "year" represents the estimated change per year in the odds of benzodiazepine prescribing, as a combination of between- and within-registrar effects.

Covariates were selected for inclusion in the multivariable model by screening each covariate for association with the outcome, using two separate models: (1) univariate - outcome vs covariate, (2) univariate (longitudinal) - outcome vs covariate + time.

"Within-Registrar" Changes in Benzodiazepine Prescribing and Initiation During Training. To assess the effect of time on individual registrars' benzodiazepine prescribing and initiation, conditional logistic regression was used with registrar included as a fixed effect to assess within-registrar changes in benzodiazepine-prescribing rates over time. The model included a time component, calculated from the registrar's term (Terms 1, 2, 3, or 4).

Covariates were selected for inclusion in the multivariable regression models by screening each covariate for association with the outcome, using two separate random effects models: (1) univariate, (2) longitudinal, with two terms in each model-covariate and time.

The covariates "Australian qualifications," "registrar gender," and "RTP" were constant within registrar (do not change within registrar over time). To assess whether these factors influenced benzodiazepine-prescribing practices over time, we included a term representing the interaction between each factor and time in models where either constant factor was included. The interaction term assesses between-registrar changes in the outcome over time. If the interaction term was not significant at $p<0.05$, both the interaction term and the constant factor were not included in the final multivariable model.

In all multivariable models (within-program temporal change and within-registrar change-for both prescribing and initiation), covariates which had $p$ values $<0.20$ in at least one of the screening univariate models were considered for inclusion in the multivariable analyses. Covariates which had a small effect size and were no longer significant (at $p<0.05$ ) in the multivariable model were tested for removal from the model. If the covariate's removal did not substantively change the resulting model, the covariate was not included in the final multivariable model.

All analyses were programmed using STATA 13.1 and SAS V9.4.

\section{Ethics}

The ReCEnT project has approval from the University of Newcastle Human Research Ethics Committee, Reference H-2009-0323. 


\section{RESULTS}

A total of 1161 individual registrars (response rate 95.5\%) contributed 2812 registrar rounds of data (including details of 136,809 individual consultations and 221,358 problems/ diagnoses in patients aged 16 or older).

The demographics of the participating registrars and practices are presented in Table 1.

\section{Frequency of Benzodiazepine Prescribing}

Benzodiazepines were prescribed in 2598 (1.9\% [95\% CI, 1.83$1.97 \%]$ ) consultations. Benzodiazepines were initiated in 702 (0.5\% [95\% CI 0.48-0.6\%]) consultations (excluding those consultations where continuing benzodiazepines were prescribed). Further analysis was at the level of problem/diagnosis. Of all problems/diagnoses $(n=221,358)$, in $2632(1.2 \%$ [95\% CI $1.1-$ $1.2 \%]$ ), a benzodiazepine was prescribed, and in $708(0.3 \% 95 \%$ CI [0.3-0.4\%] of problems/diagnoses (excluding problems/ diagnoses for which a continuing benzodiazepine prescription was provided), a benzodiazepine was initiated.

The most commonly prescribed benzodiazepines were diazepam (44.6\% of all benzodiazepines), temazepam (29.1\%), and oxazepam $(10.1 \%)$. The conditions for which benzodiazepines were most commonly prescribed (apart from "prescription renewal" as reason for prescription) were insomnia/sleep problem

Table 1 Characteristics of Participating GP Registrars and Practices

\begin{tabular}{|c|c|c|}
\hline Variable & Class & $\begin{array}{l}n(\%) \text { or } \\
\text { mean (SD) }\end{array}$ \\
\hline \multicolumn{3}{|c|}{ Registrar variables $(n=1161)^{*}$} \\
\hline \multirow[t]{2}{*}{ Registrar gender } & Male & $391(33.7)$ \\
\hline & Female & $770(66.3)$ \\
\hline \multirow{2}{*}{$\begin{array}{l}\text { Pathway registrar } \\
\text { enrolled in }\end{array}$} & General & $841(72.8)$ \\
\hline & Rural & $315(27.2)$ \\
\hline \multirow{2}{*}{$\begin{array}{l}\text { Graduated as a doctor } \\
\text { in Australia }\end{array}$} & No & $230(20.0)$ \\
\hline & Yes & $920(80.0)$ \\
\hline \multirow{4}{*}{$\begin{array}{l}\text { Number of rounds of } \\
\text { data collected }\end{array}$} & 1 & $166(14.3)$ \\
\hline & 2 & $383(33.0)$ \\
\hline & 3 & $568(48.9)$ \\
\hline & 4 & $44(3.8)$ \\
\hline \multicolumn{3}{|c|}{ Registrar-term or practice-term variables $(n=2812)^{\ddagger}$} \\
\hline Registrar age (years) & Mean (SD) & $32.7(6.4)$ \\
\hline \multirow[t]{4}{*}{ Registrar training term } & Term 1 & $1062(37.8)$ \\
\hline & Term 2 & $983(35.0)$ \\
\hline & Term 3 & $691(24.6)$ \\
\hline & Term 4 & $76(2.7)$ \\
\hline \multirow[t]{2}{*}{ Registrar works fulltime } & No & $610(22.2)$ \\
\hline & Yes & $2140(77.8)$ \\
\hline \multirow{7}{*}{$\begin{array}{l}\text { Does the practice } \\
\text { routinely bulk bill } \\
\text { Number of GPs working } \\
\text { at the practice } \\
\text { Rurality of practice }\end{array}$} & No & $2295(82.1)$ \\
\hline & Yes & $500(17.9)$ \\
\hline & 1-5 (small practice) & $940(34.3)$ \\
\hline & $6-10+$ (large practice) & $1804(65.7)$ \\
\hline & Major city & $1594(56.7)$ \\
\hline & Inner regional & $735(26.2)$ \\
\hline & $\begin{array}{l}\text { Outer regional, remote, } \\
\text { or very remote }\end{array}$ & $481(17.1)$ \\
\hline $\begin{array}{l}\text { SEIFA }^{\S} \text { Index (decile) } \\
\text { of practice }\end{array}$ & Mean (SD) & $2.9(5.5)$ \\
\hline
\end{tabular}

${ }^{*}$ Registrars enrolled in the Rural Pathway must complete all their GP terms in rural areas

${ }^{\top}$ Numbers may not add up to 1161 due to missing data

${ }^{7}$ Numbers may not add up to 2812 due to missing data

${ }^{\S}$ Socioeconomic Index for Area. Index of disadvantage
(26.7\% of all benzodiazepines), anxiety (20.6\%), and back or neck pain (5.3\%). See Table 2 for individual benzodiazepines prescribed and the conditions for which they were prescribed.

\section{Temporal Changes in Benzodiazepine Prescribing and Initiation Within the Training Program (Within Program)}

Univariate Associations of Temporal Changes in Benzodiazepine Prescribing and Initiation. In the primary and secondary analyses (of all benzodiazepine prescribing and of benzodiazepine initiation), results of the two covariate selection models showed good consistency across all covariates. The univariate associations of benzodiazepine prescribing and of benzodiazepine initiation are presented in online Appendix Table 1. In a univariate model including only the term for time, there was a significant decrease in benzodiazepine prescribing over time $(p=0.014, \mathrm{OR}=0.96$, $\mathrm{CI}=0.93,0.99)$. For benzodiazepine initiation, there was no significant change $(p=0.40, \mathrm{OR}=0.97, \mathrm{CI}=0.92,1.03)$.

Multivariable Associations of Temporal Changes in Benzodiazepine Prescribing and Initiation. Table 3 shows results from the final multivariable model with outcome "benzodiazepine prescribed." There was a significant reduction in benzodiazepine prescribing over time $(p=<0.001$, $\mathrm{OR}=0.94, \mathrm{CI}=0.90,0.97$; representing a $6 \%$ per year reduction). Figure 1 shows benzodiazepine prescribing year by year. Table 4 shows results from the final multivariable model with outcome "benzodiazepine initiated." The decrease in benzodiazepine initiation over time was not significant $(p=0.11, \mathrm{OR}=0.95[\mathrm{CI}=0.89,1.01])$.

\section{Within-Registrar Changes in Benzodiazepine Prescribing and Initiation Within the Training Program}

Univariate Associations of Within-Registrar Benzodiazepine Prescribing and Initiation. In the primary and secondary analyses of all benzodiazepine prescribing and benzodiazepine initiation, the results of the two covariate selection models showed a high (benzodiazepine-prescribing model) or good (benzodiazepine initiation model) level of consistency across all covariates. The univariate associations of benzodiazepine prescribing and of benzodiazepine initiation are presented in online Appendix Table 2. In a univariate model including only the term for time (Term), there was a significant decrease in benzodiazepine prescribing over time $(p=0.043$, OR $=0.95$, $\mathrm{CI}=0.90,1.00)$. For benzodiazepine initiation, there was no significant change $(p=0.94, \mathrm{OR}=1.00, \mathrm{CI}=0.90,1.10)$.

Multivariable Associations of Within-Registrar Benzodiazepine Prescribing and Initiation. Table 5 shows results from the final multivariable model with outcome "benzodiazepine prescribed." There was no significant change in benzodiazepine prescribing during training $(p=0.92$, 
Table 2 Most Frequent Benzodiazepine and Related Drugs Prescribed and Initiated, and Most Common Conditions for which Benzodiazepines and Related Drugs Were Prescribed. Conditions are derived by collapsing of congruent ICPC-2 codes

\begin{tabular}{|c|c|c|c|c|c|}
\hline Benzodiazepine prescribed & Freq. (\%) & Benzodiazepine initiated & Freq. (\%) & Condition & Freq. (\%) \\
\hline Diazepam & $1266(44.6)$ & Diazepam & $356(47.6)$ & Insomnia/sleep problem & $702(26.7)$ \\
\hline Temazepam & $826(29.1)$ & Temazepam & $279(37.3)$ & Anxiety & $541(20.6)$ \\
\hline Oxazepam & $286(10.1)$ & Oxazepam & $23(3.1)$ & Prescription renewal & $359(13.7)$ \\
\hline Alprazolam & $128(4.5)$ & Zolpidem & $23(3.1)$ & Back or neck pain & $140(5.3)$ \\
\hline Zolpidem & $118(4.2)$ & Zopiclone & $17(2.3)$ & Anxiety with depression & $96(3.6)$ \\
\hline Zopiclone & $67(2.4)$ & Alprazolam & 12 (1.6) & Depression & $88(3.3)$ \\
\hline Nitrazepam & $55(1.9)$ & Lorazepam & $10(1.3)$ & Dependence/addiction & $79(3.0)$ \\
\hline Lorazepam & $38(1.3)$ & Valerian & $10(1.3)$ & Panic attacks/disorder & $42(1.6)$ \\
\hline Valerian & $16(0.6)$ & Nitrazepam & $9(1.2)$ & Chronic alcohol abuse/dependence & $40(1.5)$ \\
\hline
\end{tabular}

$\mathrm{OR}=1.00, \mathrm{CI}=0.94,1.06)$. Table 6 shows results from the final multivariable model with outcome "benzodiazepine initiated." There was no significant change in benzodiazepine initiation during training $(p=0.69, \mathrm{OR}=$ $1.04[\mathrm{CI}=0.87,1.24])$.

\section{DISCUSSION}

\section{Summary of Main Findings}

We found benzodiazepine prescribing by registrars to be frequent (in $1.9 \%$ of consultations and for $1.2 \%$ of problems/ diagnoses dealt with). Initiation of benzodiazepines was less common $(0.3 \%$ of problems/diagnoses).

While overall there was a significant reduction in benzodiazepine prescribing in our training program over time (by year) on multivariable analysis, there was no significant reduction in within-registrar prescribing over the course of their training. There was no significant reduction in initiation of benzodiazepines on multivariable analysis in either by-year or within-registrar analyses.

\section{Comparison with Other Studies}

We are unaware of previous studies in primary care trainees. Internationally, studies of overall trends in benzodiazepine prescribing are not consistent. For example, there has been decreased benzodiazepine prescribing from 2002 to 2013 in Ontario, Canada, ${ }^{33}$ and a modest decrease in initiation (but not in continuing prescriptions) of benzodiazepines in France from 2006 to $2014 .^{34}$ In the USA, however, there has been an increase in ongoing benzodiazepine prescriptions from 2005 to $2012^{35}$ and an increase in primary care benzodiazepine prescriptions for older patients from 2003 to 2012 . $^{36}$

In Australia, however, there is good evidence for decreasing benzodiazepine prescribing. There was a modest reduction in prescribing (measured by defined daily doses) from 1998 to $2011{ }^{37}$ Both incidence and prevalence of benzodiazepine prescribing in Australia decreased (by 41.2 and 18.2\%, respectively) from 2007 to $2015^{38}$ — a period and setting of direct relevance to interpretation of our study findings in GP registrars.

\section{Strengths and Limitations}

Our longitudinal methodology, unlike previous cross-sectional analyses, allows confidence that we have measured changes in

Table 3 Associations with Benzodiazepines Prescriptions Over Time (Year)—Within Program: Univariate and Multivariable Models

\begin{tabular}{|c|c|c|c|c|c|}
\hline \multirow[b]{2}{*}{ Variable } & \multirow[b]{2}{*}{ Class } & \multicolumn{2}{|l|}{ Univariate } & \multicolumn{2}{|l|}{ Adjusted } \\
\hline & & OR $(95 \%$ CI $)$ & $p$ & OR $(95 \% \mathrm{CI})$ & $p$ \\
\hline Time (year) & & $0.96(0.93,0.99)$ & 0.014 & $0.94(0.90,0.97)$ & 0.001 \\
\hline Patient age group & $35-64$ & $1.77(1.59,1.97)$ & $<0.001$ & $1.60(1.42,1.81)$ & $<0.001$ \\
\hline Referent: $16-34$ & $65+$ & $1.62(1.44,1.83)$ & $<0.001$ & $1.46(1.27,1.68)$ & $<0.001$ \\
\hline Aboriginal or Torres Strait Islander & Yes & $1.75(1.36,2.27)$ & $<0.001$ & $2.00(1.53,2.60)$ & $<0.001$ \\
\hline NESB* & Yes & $0.49(0.39,0.63)$ & $<0.001$ & $0.50(0.39,0.65)$ & $<0.001$ \\
\hline Patient/practice status & New to practice & $0.76(0.63,0.92)$ & 0.004 & $1.14(0.92,1.40)$ & 0.24 \\
\hline Referent: existing patient & New to registrar & $1.00(0.92,1.08)$ & 0.99 & $1.27(1.15,1.39)$ & $<0.001$ \\
\hline Registrar gender & Female & $0.82(0.74,0.91)$ & $<0.001$ & $0.84(0.75,0.93)$ & 0.002 \\
\hline Practice routinely bulk bills & Yes & $0.87(0.77,1.00)$ & 0.044 & $0.90(0.78,1.04)$ & 0.14 \\
\hline $\mathrm{RTP}^{\dagger}$ & RTP 2 & $1.38(1.17,1.63)$ & $<0.001$ & $1.40(1.18,1.67)$ & $<0.001$ \\
\hline \multirow[t]{3}{*}{ Referent RTP 1} & RTP 3 & $1.33(1.11,1.58)$ & 0.002 & $1.34(1.11,1.62)$ & 0.0023 \\
\hline & RTP 4 & $1.24(1.09,1.41)$ & 0.001 & $1.35(1.18,1.55)$ & $<0.001$ \\
\hline & RTP 5 & $1.74(1.41,2.14)$ & $<0.001$ & $1.60(1.25,2.06)$ & $<0.001$ \\
\hline New problem & Yes & $0.25(0.22,0.27)$ & $<0.001$ & $0.21(0.18,0.23)$ & $<0.001$ \\
\hline Chronic disease & Yes & $0.82(0.74,0.91)$ & $<0.001$ & $0.51(0.45,0.57)$ & $<0.001$ \\
\hline Consultation duration & mean (SD) & $1.01(1.00,1.01)$ & 0.006 & $1.01(1.01,1.01)$ & $<0.001$ \\
\hline
\end{tabular}

*NESB, non-English-speaking background

${ }^{\dagger} R T P$, regional training provider

For each minute of consultation time 


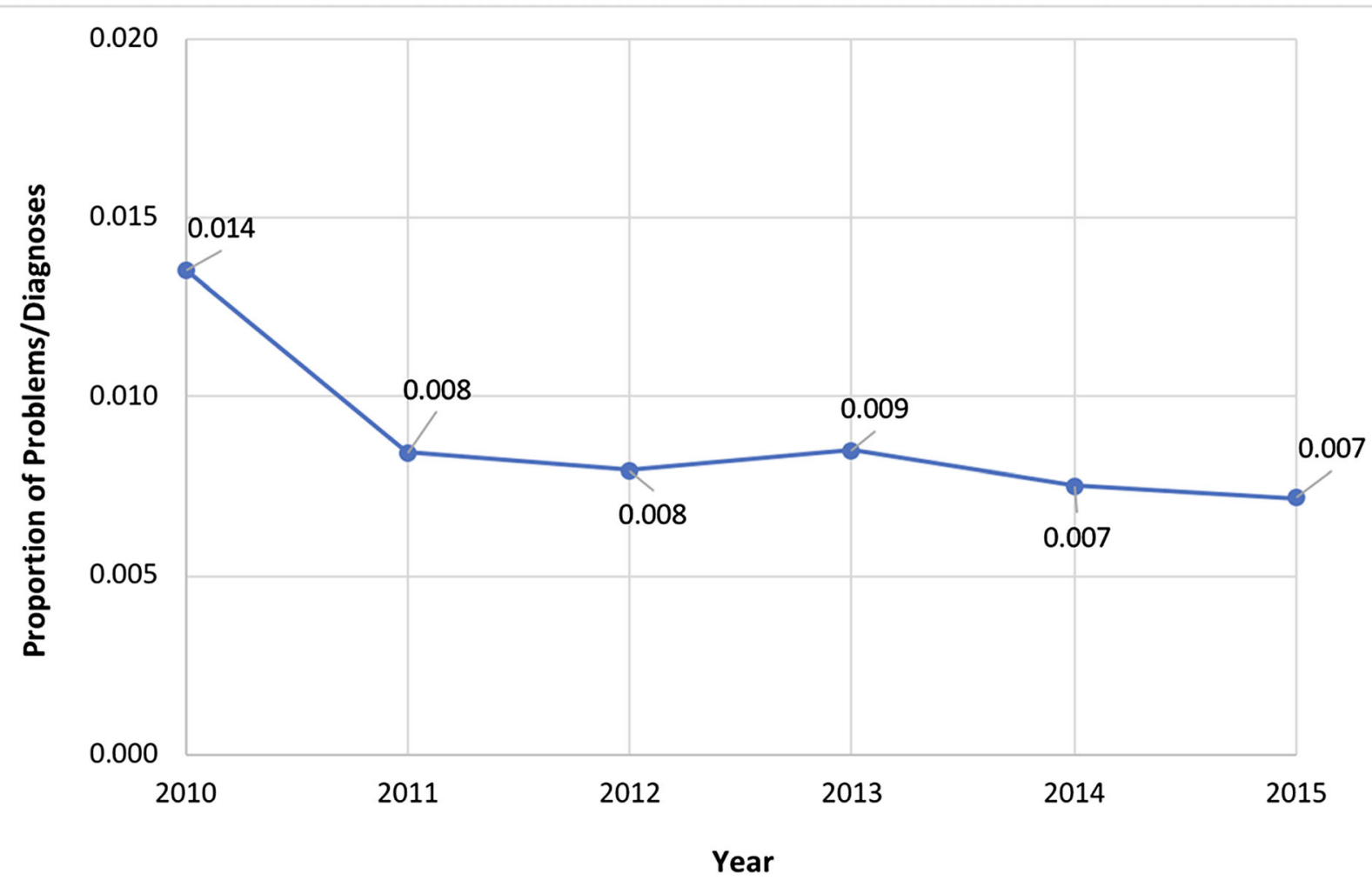

Fig. 1 Proportions of all problems/diagnoses in which a benzodiazepine or related drug was prescribed, by year (adjusted for covariates).

prescribing and initiation of benzodiazepines during earlycareer/vocational training in general practice. These can be interpreted as reflecting effects (or lack of effect) of the training environment (practice) and/or educational program on registrars' prescribing behaviors.

The large number of registrars providing detailed contemporaneous records of their individual clinical consultations provided us with adequate data to adjust for multiple demographic, clinical, and educational variables in our analysis. The response rate is particularly high for studies of GPs, ${ }^{39}$ enhancing our study's internal validity. The study population from five of 17 regional vocational training providers (across five of Australia's six states and all degrees of rurality/ urbanicity) also provides good generalizability to the Australian GP training program.

A limitation of our study is that we do not have data on appropriateness or inappropriateness of individual prescriptions. While we have the registrars' diagnosis of each problem/ diagnosis encountered, and its demographic associations, we do not have further clinical contextual data. For example, we

Table 4 Associations with Benzodiazepines Initiation Over Time (Year)—Within Program: Univariate and Multivariable Models

\begin{tabular}{|c|c|c|c|c|c|}
\hline \multirow[b]{2}{*}{ Variable } & \multirow[b]{2}{*}{ Class } & \multicolumn{2}{|l|}{ Univariate } & \multicolumn{2}{|l|}{ Adjusted } \\
\hline & & OR $(95 \% \mathrm{CI})$ & $p$ & OR $(95 \% \mathrm{CI})$ & $p$ \\
\hline Time (year) & & $0.97(0.92,1.03)$ & 0.40 & $0.95(0.89,1.01)$ & 0.11 \\
\hline Patient age group & $35-64$ & $1.26(1.06,1.50)$ & 0.009 & $1.18(0.97,1.44)$ & 0.10 \\
\hline Referent: $16-34$ & $65+$ & $0.66(0.52,0.85)$ & 0.001 & $0.65(0.49,0.86)$ & 0.002 \\
\hline Aboriginal or Torres Strait Islander & Yes & $2.65(1.75,4.01)$ & $<0.001$ & $2.30(1.48,3.56)$ & $<0.001$ \\
\hline NESB* & Yes & $0.44(0.29,0.67)$ & 0.001 & $0.47(0.30,0.72)$ & 0.001 \\
\hline Registrar gender & Female & $0.71(0.60,0.85)$ & $<0.001$ & $0.67(0.55,0.81)$ & $<0.001$ \\
\hline $\mathrm{RTP}^{\dagger}$ & RTP 2 & $0.97(0.72,1.30)$ & 0.83 & $0.98(0.73,1.32)$ & 0.89 \\
\hline \multirow[t]{3}{*}{ Referent: RTP1 } & RTP 3 & $0.96(0.72,1.27)$ & 0.77 & $0.89(0.65,1.21)$ & 0.46 \\
\hline & RTP 4 & $0.98(0.79,1.21)$ & 0.82 & $0.99(0.79,1.26)$ & 0.97 \\
\hline & RTP 5 & $1.91(1.37,2.68)$ & $<0.001$ & $1.64(1.10,2.46)$ & 0.016 \\
\hline New problem & Yes & $1.13(0.97,1.32)$ & 0.12 & $1.11(0.93,1.32)$ & 0.24 \\
\hline Sought assistance & Yes & $1.51(1.25,1.83)$ & $<0.001$ & $1.27(1.02,1.58)$ & 0.031 \\
\hline Registrar age & Mean (SD) & $1.01(1.00,1.03)$ & 0.038 & $1.01(1.00,1.03)$ & 0.054 \\
\hline Consultation duration & Mean (SD) & $1.03(1.02,1.04)$ & $<0.001$ & $1.03(1.02,1.04)$ & $<0.001$ \\
\hline
\end{tabular}

*NESB non-English-speaking background

${ }^{\dagger} R T P$ regional training provider

${ }^{\sharp}$ For each minute of consultation time 
Table 5 Associations of Within-Registrar Changes in Benzodiazepine Prescribing (By Term): Univariate and Multivariable Models

\begin{tabular}{|c|c|c|c|c|c|}
\hline \multirow[b]{2}{*}{ Variable } & \multirow[b]{2}{*}{ Class } & \multicolumn{2}{|c|}{ Univariate (including time) } & \multicolumn{2}{|l|}{ Adjusted } \\
\hline & & OR (95\% CI) & $p$ & OR $(95 \% \mathrm{CI})$ & $p$ \\
\hline Time (term) & & $0.95(0.90,1.00)$ & 0.043 & $1.00(0.94,1.06)$ & 0.92 \\
\hline Patient age group & $35-64$ & $1.77(1.60,1.97)$ & $<0.001$ & $1.59(1.42,1.78)$ & $<0.001$ \\
\hline Referent: $16-34$ & $65+$ & $1.63(1.44,1.84)$ & $<0.001$ & $1.44(1.25,1.65)$ & $<0.001$ \\
\hline Aboriginal or Torres Strait Islander & Yes & $1.74(1.35,2.25)$ & $<0.001$ & $1.96(1.47,2.62)$ & $<0.001$ \\
\hline NESB* & Yes & $0.49(0.38,0.63)$ & $<0.001$ & $0.51(0.40,0.66)$ & $<0.001$ \\
\hline Patient/practice status & New to practice & $0.76(0.63,0.91)$ & 0.003 & $1.13(0.91,1.40)$ & 0.27 \\
\hline Referent: existing patient & New to registrar & $0.99(0.91,1.08)$ & 0.84 & $1.28(1.16,1.40)$ & $<0.001$ \\
\hline Practice routinely bulk bills & Yes & $0.87(0.76,0.99)$ & 0.037 & $0.87(0.72,1.05)$ & 0.14 \\
\hline New problem & Yes & $0.24(0.22,0.27)$ & $<0.001$ & $0.21(0.18,0.23)$ & $<0.001$ \\
\hline Chronic disease & Yes & $0.83(0.75,0.91)$ & $<0.001$ & $0.51(0.46,0.57)$ & $<0.001$ \\
\hline Consultation duration $^{\dagger}$ & & $1.01(1.00,1.01)$ & 0.011 & $1.01(1.01,1.02)$ & $<0.001$ \\
\hline
\end{tabular}

The covariate "sought assistance" was no longer significant and had a small effect size in the multivariable model, and so was tested for removal from the model. Its removal did not alter the model so this covariate was not included in the multivariable model

*NESB non-English-speaking background

${ }^{\dagger}$ For each minute of consultation time

have data only on medications prescribed in the index consultation - we do not have data on medicine regimens (including opioids or other medicines that may be problematic in those using benzodiazepines). Similarly, while we have tightly linked data on the problem/diagnosis for which the benzodiazepine was prescribed, we do not have data on co-morbidities not addressed at the index consultation (for example, alcohol or other drug use). We interpret our findings, however, in the context of very limited indications for benzodiazepine use in contemporary practice $^{2}$ and of general acknowledgement of over-prescription of benzodiazepines as a major health problem in Australia. ${ }^{40} \mathrm{~A}$ further limitation is the small number of consultations in (the non-compulsory) Term 4.

\section{Implications for Clinical and Educational Practice}

Our findings are of clinically as well as statistically significant year-by-year reductions in benzodiazepine prescribing (6\% per year). The disparity between these overall training program changes and within-registrar changes in prescribing may reflect the primacy of the practicebased "apprenticeship-like" component of GP vocational training. ${ }^{25}$
Our previous research in this registrar population ${ }^{41,42}$ has suggested that prescribing patterns of GPs within the registrar's practice and the practice-based "apprenticeship" model of training can be determinants of antibiotic prescribing. Furthermore, an educational intervention that concurrently targeted registrars and supervisors ${ }^{43}$ produced a significant reduction in registrars' inappropriate antibiotic prescribing for acute bronchitis. ${ }^{44}$

Thus, the temporal trend within the training program for registrars as a whole to prescribe fewer benzodiazepines year by year appears to reflect decreasing benzodiazepine prescription within the wider clinical environment ${ }^{38}$ in which the apprenticeship model of training sits. This may plausibly be due to the incremental effect of continuing educational efforts to reduce benzodiazepine prescribing in the Australian GP population by organizations such as NPS MedicineWise and the Royal Australian College of General Practitioners. ${ }^{40}$

A lack of reduction "within-registrars" as they progress through training may suggest a lack of effect of any current specific in-training education around the inappropriateness of most benzodiazepine prescribing. This specific in-training education may be delivered centrally by training organizations and in one-on-one scheduled weekly education sessions between registrars and their accredited supervisor (an

Table 6 Associations of Within-Registrar Changes in Benzodiazepine Initiation (By Term): Univariate and Multivariable Models

\begin{tabular}{|c|c|c|c|c|c|}
\hline \multirow[b]{2}{*}{ Variable } & \multirow[b]{2}{*}{ Class } & \multicolumn{2}{|c|}{ Univariate (including time) } & \multicolumn{2}{|l|}{ Adjusted } \\
\hline & & OR $(95 \%$ CI $)$ & $p$ & OR (95\% CI) & $p$ \\
\hline Time (term) & & $1.00(0.90,1.10)$ & 0.94 & $1.04(0.87,1.24)$ & 0.69 \\
\hline Patient age group & $35-64$ & $1.26(1.06,1.51)$ & 0.009 & $1.18(0.98,1.43)$ & 0.085 \\
\hline Referent: $16-34$ & $65+$ & $0.67(0.52,0.85)$ & 0.001 & $0.68(0.52,0.90)$ & 0.007 \\
\hline Aboriginal or Torres Strait Islander & Yes & $2.63(1.74,3.98)$ & $<0.001$ & $2.39(1.50,3.81)$ & $<0.001$ \\
\hline NESB* & Yes & $0.44(0.29,0.67)$ & 0.001 & $0.49(0.30,0.78)$ & 0.003 \\
\hline New problem & Yes & $1.13(0.97,1.32)$ & 0.12 & $1.07(0.91,1.27)$ & 0.40 \\
\hline Sought assistance & Yes & $1.52(1.25,1.84)$ & $<0.001$ & $1.32(1.05,1.65)$ & 0.016 \\
\hline Registrar age & & $1.02(1.00,1.03)$ & 0.031 & $0.99(0.76,1.28)$ & 0.94 \\
\hline Consultation duration ${ }^{\dagger}$ & & $1.03(1.02,1.04)$ & $<0.001$ & $1.03(1.02,1.04)$ & $<0.001$ \\
\hline
\end{tabular}

*NESB non-English-speaking background

${ }^{\dagger}$ For each minute of consultation time 
experienced GP). This is of importance as, despite welcome temporal trends to less benzodiazepine prescribing in this and other Australian studies, inappropriate benzodiazepine use remains a major health issue. ${ }^{45}$

Effective educational strategies for this topic should be prioritized. Joint or concurrent education of the registrarsupervisor dyad specifically addressing rational prescribing and barriers to discontinuation (e.g., teaching non-pharmacological management techniques, not just focusing on benzodiazepine harms education) and specifically seeking to change clinical behavior as well as knowledge is a potential approach. The efficacy of this approach has been demonstrated for other inappropriate prescribing (antibiotics for acute bronchitis) in this population. ${ }^{43}, 44,46$

\section{CONCLUSIONS}

Despite a welcome temporal trend for benzodiazepineprescribing reduction from 2010 to 2015, this remains an area for rational prescribing improvement. Our findings suggest an opportunity for targeted education of registrars and their supervisors.

Acknowledgements: The authors would like to thank the registrars and practices who participated in ReCEnT.

Corresponding Author: Parker Magin, PhD, FRACGP; Discipline of General Practice, University of Newcastle, University Drive, Callaghan, NSW 2308, Australia (e-mail: parker.magin@newcastle.edu.au).

Funders During the data collection period included in this study, funding of the ReCEnT study was by the participating educational organizations: General Practice Training Valley to Coast, the Victorian Metropolitan Alliance, General Practice Training Tasmania, Tropical Medical Training, and Adelaide to Outback GP Training Program. These organizations were funded by the Australian Department of Health. Since 2016, the ReCEnT study is funded by an Australian Commonwealth Department of Health Research Grant and supported by GP Synergy, the general practice Regional Training Organization for New South Wales and the Australian Capital Territory. GP Synergy is funded by the Australian Department of Health.

\section{Compliance with Ethical Standards:}

Ethics: The ReCEnT project has approval from the University of Newcastle Human Research Ethics Committee, Reference H-2009-0323.

Conflict of Interest: The authors declare that they have no conflict of interest.

\section{REFERENCES}

1. Donoghue $\mathbf{J}$, Lader $\mathbf{M}$. Usage of benzodiazepines: A review. Int $\mathrm{J} \mathrm{J}$ Psychiatry ClinPract 2010;14:78-87.

2. Psychotropic Expert Groups. Therapeutic guidelines: Psychotropic. Version 7. Melbourne: Therapeutic Guidelines Limited. 2013.

3. Gaseem A, Kansagara D, Forciea MA, Cooke M, Denberg TD, Clinical Guidelines Committee of the American College of P. Management of Chronic Insomnia Disorder in Adults: A Clinical Practice Guideline From the American College of Physicians. Ann Intern Med 2016;165: 125-33.
4. Glass J, Lanctot KL, Herrmann N, Sproule BA, Busto UE. Sedative hypnotics in older people with insomnia: meta-analysis of risks and benefits. BMJ 2005;331:1169.

5. Bourgeois J, Elseviers MM, Van Bortel L, Petrovic M, Vander Stichele RH. Sleep quality of benzodiazepine users in nursing homes: a comparative study with nonusers. Sleep Med 2013;14:614-21.

6. Billioti de Gage S, Moride $\mathbf{Y}$, Ducruet $\mathbf{T}$, et al. Benzodiazepine use and risk of Alzheimer's disease: case-control study. BMJ 2014;349:g5205.

7. Benard-Laribiere A, Noize $\mathbf{P}$, Pambrun E, et al. Comorbidities and concurrent medications increasing the risk of adverse drug reactions: prevalence in French benzodiazepine users. Eur J Clin Pharmacol 2016;72:869-76.

8. Taipale $\mathbf{H}$, Tolppanen A-M, Koponen $\mathbf{M}$, et al. Risk of pneumonia associated with incident benzodiazepine use among community-dwelling adults with Alzheimer disease. CMAJ 2017;189:E519-E29.

9. Hood SD, Norman A, Hince DA, Melichar JK, Hulse GK. Benzodiazepine dependence and its treatment with low dose flumazenil. $\mathrm{Br} \mathrm{J}$ Clin Pharmacol 2014;77:285-94.

10. Parsaik AK, Mascarenhas SS, Khosh-Chashm D, et al. Mortality associated with anxiolytic and hypnotic drugs-A systematic review and meta-analysis. Aust N Z J Psychiatry 2016;50:520-33.

11. Wilt TJ, MacDonald R, Brasure M, et al. Pharmacologic Treatment of Insomnia Disorder: An Evidence Report for a Clinical Practice Guideline by the American College of Physicians. Ann Intern Med 2016;165:103-12.

12. Gerlach LB, Olfson M, Kales HC, Maust DT. Opioids and Other Centra Nervous System-Active Polypharmacy in Older Adults in the United States. J Am Geriatr Soc 2017;65:2052-6.

13. Hwang CS, Kang EM, Kornegay CJ, Staffa JA, Jones CM, McAninch JK. Trends in the Concomitant Prescribing of Opioids and Benzodiazepines, 2002-2014. Am J Prev Med 2016;51:151-60.

14. Karaca-Mandic P, Meara E, Morden NE. The growing problem of cotreatment with opioids and benzodiazepines. BMJ 2017;356:j1224.

15. Sun EC, Dixit A, Humphreys K, Darnall BD, Baker LC, Mackey S. Association between concurrent use of prescription opioids and benzodiazepines and overdose: retrospective analysis. BMJ 2017;356:j760.

16. Weymann D, Gladstone EJ, Smolina K, Morgan SG. Long-term sedative use among community-dwelling adults: a population-based analysis. CMAJ Open 2017;5:E52-E60.

17. Kroll DS, Nieva HR, Barsky AJ, Linder JA. Benzodiazepines are Prescribed More Frequently to Patients Already at Risk for Benzodiazepine-Related Adverse Events in Primary Care. J Gen Intern Med 2016;31:1027-34.

18. Morgan SG, Weymann D. Patterns, predictors and persistence of chronic sedative use: a population-based observational study of older adults in British Columbia, Canada. Eur J Clin Pharmacol 2017;73:1001-8.

19. Lucchetti G, Lucchetti ALG. Inappropriate prescribing in older persons: A systematic review of medications available in different criteria. Arch Gerontol Geriatr 2017;68:55-61.

20. Jorm AF, Grayson D, Creasey H, Waite L, Broe GA. Long-term benzodiazepine use by elderly people living in the community. Aust $\mathrm{N} \mathrm{Z}$ J Public Health 2000;24:7-10.

21. Bjornsdottir I, Kristinsson KG, Hansen EH. Diagnosing infections: a qualitative view on prescription decisions in general practice over time. Pharmacy World \& Science 2010;32:805-14.

22. Holliday SM, Morgan S, Tapley A, et al. The pattern of anxiolytic and hypnotic management by Australian general practice trainees. Drug Alcohol Rev 2017;36:261-9.

23. Emery JD, Skinner LP, Morgan S, Guest BJ, Vickery AW. Future models of general practice training in Australia. Med $\mathrm{J}$ Aust 2011;194:S97-100.

24. Hays RB, Morgan S. Australian and overseas models of general practice training. Med J Aust 2011;194:S63-4.

25. Thomson JS, Anderson KJ, Mara PR, Stevenson AD. Supervisiongrowing and building a sustainable general practice supervisor system. Med J Aust 2011;194:S101-4.

26. Royal Australian College of General Practitioners Curriculum. 2011. RACGP. Melbourne.

27. Morgan S, Magin PJ, Henderson KM, et al. Study protocol: the Registrar Clinical Encounters in Training (ReCEnT) study. BMC Fam Pract 2012;13:50.

28. Ferracioli-Oda E, Qawasmi A, Bloch MH. Meta-analysis: melatonin for the treatment of primary sleep disorders. PLoS ONE 2013;8:e63773.

29. Australian Bureau of Statistics. Australian Standard Geographical Classification (ASGC) - 2006. at http://www.abs.gov.au/AUSSTATS/ 
abs@.nsf/Latestproducts/1AE106C101420508CA2571A900170741 : (accessed 11/6/18). 2006

30. Australian Bureau of Statistics. 2039.0 - Information Paper: An Introduction to Socio-economic Indexes of Areas (SEIFA), 2006. Available at: http:// www.abs.gov.au/ausstats/abs@.nsf/mf/2039.0/ [accessed 11/6/18].

31. O'Halloran J, Miller GC, Britt H. Defining chronic conditions for primary care with ICPC-2. Fam Pract 2004;21:381-6.

32. Britt $\mathbf{H}$. A new coding tool for computerised clinical systems in primary care-ICPC plus. Aust Fam Physician 1997;26 Suppl 2:S79-82.

33. Iaboni A, Bronskill SE, Reynolds KB, et al. Changing Pattern of Sedative Use in Older Adults: A Population-Based Cohort Study. Drugs Aging 2016;33:523-33.

34. Benard-Laribiere A, Noize $\mathbf{P}$, Pambrun E, et al. Trends in incident use of benzodiazepines and Z-drugs in France from 2006 to 2012: a populationbased study. Pharmacoepidemiol Drug Saf 2017;26:162-9.

35. Kaufmann CN, Spira AP, Depp CA, Mojtabai R. Continuing Versus New Prescriptions for Sedative-Hypnotic Medications: United States, 20052012. Am J Public Health 2016;106:2019-25.

36. Maust DT, Blow FC, Wiechers IR, Kales HC, Marcus SC. National Trends in Antidepressant, Benzodiazepine, and Other Sedative-Hypnotic Treatment of Older Adults in Psychiatric and Primary Care. J Clin Psychiatry 2017;78:e363-e71.

37. Islam MM, Conigrave KM, Day CA, Nguyen Y, Haber PS. Twenty-year trends in benzodiazepine dispensing in the Australian population. Intern Med J 2014;44:57-64.

38. Brett J, Karanges EA, Daniels B, et al. Psychotropic medication use in Australia, 2007 to 2015: Changes in annual incidence, prevalence and treatment exposure. Aust N Z J Psychiatry 2017:4867417721018.
39. Bonevski B, Magin P, Horton G, Foster M, Girgis A. Response rates in GP surveys - trialling two recruitment strategies. Aust Fam Physician 2011;40:427-30.

40. Benzodiazepine dependence: reduce the risk. NPS Medicinewise. 2015. Available at: https://www.nps.org.au/medical-info/clinical-topics / news/benzodiazepine-dependence-reduce-the-risk (accessed 11/6/18)

41. Dallas A, Magin P, Morgan S, et al. Antibiotic prescribing for respiratory infections: a cross-sectional analysis of the ReCEnT study exploring the habits of early-career doctors in primary care. Fam Pract 2015;32:49-55.

42. Dallas A, van Driel M, van de Mortel T, Magin P. Antibiotic prescribing for the future: exploring the attitudes of trainees in general practice. $\mathrm{Br} \mathrm{J}$ Gen Pract 2014;64:e561-7.

43. van Driel M, Morgan S, Tapley A, et al. Study protocol of the ChAP study: Changing the antibiotic prescribing of general practice registrars through better adherence to antibiotic guidelines. BMC Fam Pract 2016;17:67.

44. Magin P, Tapley A, Morgan S, et al. Reducing early-career general practitioners' antibiotic prescribing for respiratory tract infections: a pragmatic prospective non-randomised controlled trial. Fam Pract 2015; 35: 53-60.

45. Brett J, Murnion B. Management of benzodiazepine misuse and dependence. Aust Prescriber 2015; 38:152-5.

46. Deckx L, Anthierens S, Magin P, et al. Focus on early-career GPs: qualitative evaluation of a multi-faceted educational intervention to improve antibiotic prescribing. Fam Pract. 2018; 35:99-104. 